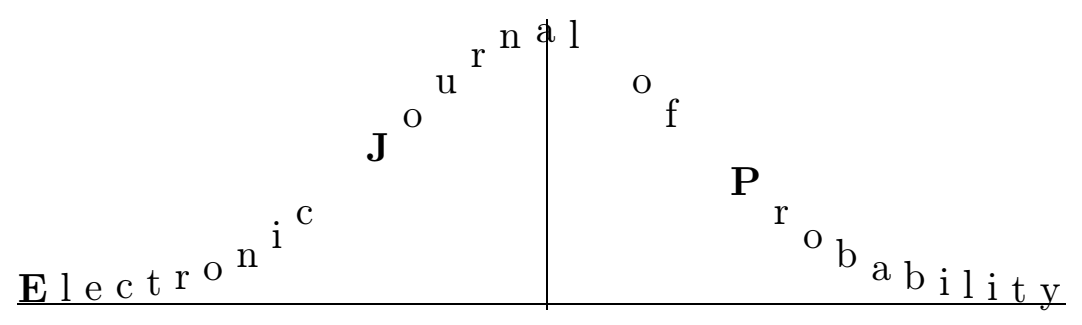

Vol. 2 (1997) Paper no. 2, pages 1-32.

Journal URL

http://www.math.washington.edu/ ejpecp/

Paper URL

http://www.math.washington.edu/ ejpecp/EjpVol2/paper2.abs.html

\title{
LAWS OF THE ITERATED LOGARITHM FOR TRIPLE INTERSECTIONS OF THREE DIMENSIONAL RANDOM WALKS
}

\section{Jay Rosen}

Department of Mathematics

College of Staten Island, CUNY

Staten Island, NY 10314

jrosen3@idt.net 
Abstact: Let $X=\left\{X_{n}, n \geq 1\right\}, X^{\prime}=\left\{X_{n}^{\prime}, n \geq 1\right\}$ and $X^{\prime \prime}=\left\{X_{n}^{\prime \prime}, n \geq\right.$ $1\}$ be three independent copies of a symmetric random walk in $Z^{3}$ with $E\left(\left|X_{1}\right|^{2} \log _{+}\left|X_{1}\right|\right)<\infty$. In this paper we study the asymptotics of $I_{n}$, the number of triple intersections up to step $n$ of the paths of $X, X^{\prime}$ and $X^{\prime \prime}$ as $n \rightarrow \infty$. Our main result is

$$
\limsup _{n \rightarrow \infty} \frac{I_{n}}{\log (n) \log _{3}(n)}=\frac{1}{\pi|Q|} \quad \text { a.s. }
$$

where $Q$ denotes the covariance matrix of $X_{1}$. A similar result holds for $J_{n}$, the number of points in the triple intersection of the ranges of $X, X^{\prime}$ and $X^{\prime \prime}$ up to step $n$.

Keywords: random walks, intersections

AMS subject classification: 60J15, 60F99

This research was supported, in part, by grants from the National Science Foundation, the U.S.-Israel Binational Science Foundation and PSC-CUNY.

Submitted to EJP on August 27, 1996. Final version accepted on March 26, 1997. 


\title{
Laws of the Iterated Logarithm for Triple Intersections of Three Dimensional Random Walks
}

\author{
Jay Rosen*
}

\begin{abstract}
Let $X=\left\{X_{n}, n \geq 1\right\}, X^{\prime}=\left\{X_{n}^{\prime}, n \geq 1\right\}$ and $X^{\prime \prime}=\left\{X_{n}^{\prime \prime}, n \geq 1\right\}$ be three independent copies of a symmetric random walk in $Z^{3}$ with $E\left(\left|X_{1}\right|^{2} \log _{+}\left|X_{1}\right|\right)<\infty$. In this paper we study the asymptotics of $I_{n}$, the number of triple intersections up to step $n$ of the paths of $X$, $X^{\prime}$ and $X^{\prime \prime}$ as $n \rightarrow \infty$. Our main result is

$$
\limsup _{n \rightarrow \infty} \frac{I_{n}}{\log (n) \log _{3}(n)}=\frac{1}{\pi|Q|} \quad \text { a.s. }
$$

where $Q$ denotes the covariance matrix of $X_{1}$. A similar result holds for $J_{n}$, the number of points in the triple intersection of the ranges of $X, X^{\prime}$ and $X^{\prime \prime}$ up to step $n$.
\end{abstract}

\section{Introduction}

Let $X=\left\{X_{n}, n \geq 1\right\}, X^{\prime}=\left\{X_{n}^{\prime}, \geq 1\right\}$, and $X^{\prime \prime}=\left\{X_{n}^{\prime \prime}, n \geq 1\right\}$ be three independent copies of a random walk in $Z^{3}$ with zero mean and finite variance. In this paper we study the asymptotics of the number of triple intersections up to step $n$ of the paths of $X, X^{\prime}$ and $X^{\prime \prime}$ as $n \rightarrow \infty$, both the number of 'intersection times'

$$
I_{n}=\sum_{i=1}^{n} \sum_{j=1}^{n} \sum_{k=1}^{n} 1_{\left\{X_{i}=X_{j}^{\prime}=X_{k}^{\prime \prime}\right\}}
$$

${ }^{*}$ This research was supported, in part, by grants from the National Science Foundation, the U.S.-Israel Binational Science Foundation and PSC-CUNY. 
and the number of 'intersection points'

$$
J_{n}=\left|X(1, n) \cap X^{\prime}(1, n) \cap X^{\prime \prime}(1, n)\right|
$$

where $X(1, n)$ denotes the range of $X$ up to time $n$ and $|A|$ denotes the cardinality of the set $A$. For random walks with finite variance, dimension three is the 'critical case' for triple intersections, since $I_{n}, J_{n} \uparrow \infty$ almost surely but three independent Brownian motions in $R^{3}$ do not intersect. This implies that in some sense $I_{n}, J_{n} \uparrow \infty$ slowly. We also note that in dimension $>3$ we have $I_{\infty}, J_{\infty}<\infty$ a.s.

We assume that $X_{n}$ is adapted, which means that $X_{n}$ does not live on any proper subgroup of $Z^{3}$. In the terminology of Spitzer [9] $X_{n}$ is aperiodic.

We have the following two limit theorems.

Theorem 1 Assume that $E\left(\left|X_{1}\right|^{2} \log _{+}\left|X_{1}\right|\right)<\infty$. Then

$$
\limsup _{n \rightarrow \infty} \frac{I_{n}}{\log (n) \log _{3}(n)}=\frac{1}{\pi|Q|} \quad \text { a.s. }
$$

where $Q$ denotes the covariance matrix of $X_{1}$.

As usual, $\log _{j}$ denotes the $j$-fold iterated logarithm.

In the particular case of the simple random walk on $Z^{3}$, where $Q=\frac{1}{3} I$, Theorem 1 states that

$$
\limsup _{n \rightarrow \infty} \frac{I_{n}}{\log (n) \log _{3}(n)}=\frac{27}{\pi} \quad \text { a.s. }
$$

A similar result holds for $J_{n}$ :

Theorem 2 Assume that $E\left(\left|X_{1}\right|^{2} \log _{+}\left|X_{1}\right|\right)<\infty$. Then

$$
\limsup _{n \rightarrow \infty} \frac{J_{n}}{\log (n) \log _{3}(n)}=\frac{q^{3}}{\pi|Q|} \quad \text { a.s. }
$$

where $q$ denotes the probability that $X$ will never return to its initial point.

Le Gall [4] proved that $(\log n)^{-1} J_{n}$ converges in distribution to a gamma random variable. This paper is an outgrowth of my paper with Michael Marcus [6] in which we prove analogous laws of the iterated logarithm for intersections of two symmetric random walks in $Z^{4}$ with finite third moment. In this paper we also use some of the ideas of [4] along with techniques developed in $[8,7]$. 


\section{Proof of Theorem 1}

We use $p_{n}(x)$ to denote the transition function for $X_{n}$. Recall

$$
\begin{aligned}
I_{n} & =\sum_{i=1}^{n} \sum_{j=1}^{n} \sum_{k=1}^{n} 1_{\left\{X_{i}=X_{j}^{\prime}=X_{k}^{\prime \prime}\right\}} \\
& =\sum_{x \in \mathbf{Z}^{3}}\left\{\left(\sum_{i=1}^{n} 1_{\left\{X_{i}=x\right\}}\right)\left(\sum_{j=1}^{n} 1_{\left\{X_{j}^{\prime}=x\right\}}\right)\left(\sum_{k=1}^{n} 1_{\left\{X_{k}^{\prime \prime}=x\right\}}\right)\right\}
\end{aligned}
$$

We set

$$
h(n)=E\left(I_{n}\right)=\sum_{x \in \mathbf{Z}^{3}}\left\{\left(\sum_{i=1}^{n} p_{i}(x)\right)\left(\sum_{j=1}^{n} p_{j}(x)\right)\left(\sum_{k=1}^{n} p_{k}(x)\right)\right\} .
$$

With

$$
u_{t}(x)=\sum_{r=1}^{t} p_{r}(x)
$$

we have

$$
h(t)=\sum_{x \in \mathbf{Z}^{3}}\left(u_{t}(x)\right)^{3} .
$$

As shown in [9] the random walk $X_{n}$ is adapted if and only if the origin is the unique element of $T^{3}$ satisfying $\phi(p)=1$ where $\phi(p)$ is the characteristic function of $X_{1}$ and $T^{3}=(-\pi, \pi]^{3}$ is the usual three dimensional torus. We use $\tau$ to denote the number of elements in the set $\left\{p \in T^{3}|| \phi(p) \mid=1\right\}$. We say that $X$ is aperiodic if $\tau=1$. (In Spitzer[9] this is called strongly aperiodic). We will prove our theorems for $X$ aperiodic, but using the ideas of section 2.4 in [5] it is then easy to show that they also hold if $\tau>1$. According to the local central limit theorem, P7.9 and P7.10 of [9],

$$
p_{n}(x)=\frac{q_{n}\left(Q^{-1 / 2} x\right)}{|Q|^{1 / 2}}+\inf \left(\frac{1}{n^{3 / 2}}, \frac{1}{|x|^{2} n^{1 / 2}}\right) o\left(1_{n}\right)
$$

where $q_{t}(x)$ denotes the transition density for Brownian motion in $R^{3}$ and $Q$ denotes the covariance matrix of $X_{1}$. Then, arguing seperately in the regions $n \leq n_{0}, n_{0}<n \leq|x|^{2}$ and $n>|x|^{2}$ we have

$$
u_{t}(x)=\sum_{n=1}^{t} \frac{q_{n}\left(Q^{-1 / 2} x\right)}{|Q|^{1 / 2}}+\frac{o\left(1_{n_{0}}\right)}{|x|}+\frac{O\left(n_{0}^{1 / 2}\right)}{|x|^{2}}
$$


Since

$$
\int_{0}^{\infty} q_{s}(x) d s=\frac{1}{2 \pi|x|}
$$

we see that taking $n_{0}=1$ in $(2.5)$ gives the bound

$$
u_{t}(x) \leq \frac{c}{1+|x|}
$$

We also have

$$
\begin{aligned}
\sum_{x \in \mathbf{Z}^{3}} & \left(\sum_{n=1}^{t} \frac{q_{n}\left(Q^{-1 / 2} x\right)}{|Q|^{1 / 2}}\right)^{3} \\
& \sim \int_{x \in \mathbf{R}^{3}}\left(\frac{\int_{s=1}^{t} q_{s}\left(Q^{-1 / 2} x\right) d s}{|Q|^{1 / 2}}\right)^{3} d x \\
& =\frac{1}{|Q|^{3 / 2}} \int_{x \in \mathbf{R}^{3}}\left(\int_{s=1}^{t} q_{s}\left(Q^{-1 / 2} x\right) d s\right)^{3} d x \\
& =\frac{1}{|Q|} \int_{x \in \mathbf{R}^{3}}\left(\int_{s=1}^{t} q_{s}(x) d s\right)^{3} d x \\
& =\frac{1}{(2 \pi)^{9 / 2}|Q|} \int_{a=1}^{t} \int_{b=1}^{t} \int_{c=1}^{t} \\
& \left.=\frac{1}{(2 \pi)^{3}|Q|} \int_{a=1}^{t} \int_{b=1}^{t} \int_{c=1}^{t} \int_{x \in \mathbf{R}^{3}} \exp \left(-\frac{|x|^{2}}{2}\left(\frac{1}{a}+\frac{1}{b}+\frac{1}{c}\right)\right) d x\right) d a d b d c \\
= & \frac{(a b c)^{-3 / 2}\left(\frac{1}{a}+\frac{1}{b}+\frac{1}{c}\right)^{-3 / 2} d a d b d c}{(2 \pi)^{3}|Q|} \int_{a=1}^{t} \int_{b=1}^{t} \int_{c=1}^{t}(b c+a c+a b)^{-3 / 2} d a d b d c
\end{aligned}
$$

Changing variables, first $x=a b, y=a c, z=b c$, and then $x=u^{2}, y=v^{2}, z=$ $w^{2}$ we have

$$
\begin{aligned}
& \int_{a=1}^{t} \int_{b=1}^{t} \int_{c=1}^{t} \frac{1}{(b c+a c+a b)^{3 / 2}} d a d b d c \\
& \quad=\iiint_{1 \leq x, y, z \leq t^{2}} \frac{1}{(x+y+z)^{3 / 2}} \frac{1}{2 \sqrt{x y z}} d x d y d z
\end{aligned}
$$




$$
\begin{aligned}
& =4 \iiint_{1 \leq u, v, w \leq t} \frac{1}{\left(u^{2}+v^{2}+w^{2}\right)^{3 / 2}} d u d v d w \\
& \sim 2 \pi^{2} \log t .
\end{aligned}
$$

Hence, taking $n_{0}$ large in (2.5), we have

$$
h(t) \sim \frac{1}{4 \pi|Q|} \log t .
$$

Thus the assertion of Theorem 1 can be written as

$$
\limsup _{n \rightarrow \infty} \frac{I_{n}}{4 h(n) \log _{2} h(n)}=1 \quad \text { a.s. }
$$

We begin our proof with some moment calculations.

$$
\begin{aligned}
E\left(I_{t}^{n}\right) & =\sum_{x_{1}, \ldots, x_{n}}\left\{E\left(\prod_{i=1}^{n} \sum_{r_{i}=1}^{t} 1_{\left\{X_{r_{i}}=x_{i}\right\}}\right)\right\}^{3} \\
& =\sum_{x_{1}, \ldots, x_{n}}\left\{\sum_{\pi} \sum_{r_{1} \leq r_{2} \leq \ldots \leq r_{n} \leq t} E\left(\prod_{i=1}^{n} 1_{\left\{X_{r_{i}}=x_{\pi(i)}\right\}}\right)\right\}^{3} \\
& =\sum_{x_{1}, \ldots, x_{n}}\left(\sum_{\pi} \sum_{r_{1} \leq r_{2} \leq \ldots \leq r_{n} \leq t} \prod_{i=1}^{n} p_{r_{i}-r_{i-1}}\left(x_{\pi(i)}-x_{\pi(i-1)}\right)\right)^{3} \\
= & n ! \sum_{x_{1}, \ldots, x_{n}}\left(\sum_{r_{1} \leq r_{2} \leq \ldots \leq r_{n} \leq t} \prod_{i=1}^{n} p_{r_{i}-r_{i-1}}\left(x_{i}-x_{i-1}\right)\right) \\
& \quad\left(\sum_{\pi} \sum_{s_{1} \leq s_{2} \leq \ldots \leq s_{n} \leq t} \prod_{j=1}^{n} p_{s_{j}-s_{j-1}}\left(x_{\pi(j)}-x_{\pi(j-1)}\right)\right)^{2}
\end{aligned}
$$

where $\sum_{\pi}$ runs over the set of permutations $\pi$ of $\{1,2, \ldots, n\}$. We see from (2.12) that

$$
\begin{aligned}
E\left(I_{t}^{n}\right) \leq n ! & \sum_{x_{1}, \ldots, x_{n}}\left(\prod_{i=1}^{n} u_{t}\left(x_{i}-x_{i-1}\right)\right) \\
& \left(\sum_{\pi} \prod_{j=1}^{n} u_{t}\left(x_{\pi(j)}-x_{\pi(j-1)}\right)\right)^{2},
\end{aligned}
$$


while

$$
\begin{aligned}
E\left(I_{t}^{n}\right) \geq n ! \sum_{x_{1}, \ldots, x_{n}} & \left(\prod_{i=1}^{n} u_{t / n}\left(x_{i}-x_{i-1}\right)\right) \\
& \left(\sum_{\pi} \prod_{j=1}^{n} u_{t / n}\left(x_{\pi(j)}-x_{\pi(j-1)}\right)\right)^{2} .
\end{aligned}
$$

Note that

$$
p_{j}(x)=P\left(X_{j}=x\right) \leq P\left(\left|X_{j}\right| \geq|x|\right) \leq \frac{C j}{|x|^{2}}
$$

so that

$$
u_{t}(x) \leq C \frac{t^{2}}{|x|^{2}}
$$

giving us the bound

$$
u_{t}(x) \leq \frac{C}{1+|x|^{3 / 2}} \text { for all }|x|>t^{4} .
$$

Lemma 1 For all integers $n, t \geq 0$ and for any $\epsilon>0$

$$
E\left(I_{t}^{n}\right) \leq(1+\epsilon) \Psi(n) h^{n}(t)+R(n, t)
$$

where

$$
0 \leq R(n, t) \leq C(n !)^{5} h^{n-1 / 3}(t)
$$

and

$$
\Psi(n)=\prod_{j=1}^{n-1}(4 j+1) .
$$

Proof of Lemma 1: We will make use of several ideas of Le Gall, [4]. We begin by rewriting $(2.13)$ as

$$
E\left(I_{t}^{n}\right) \leq n ! \sum_{y_{1}, \ldots, y_{n}}\left(\prod_{i=1}^{n} u_{t}\left(y_{i}\right)\right)\left(\sum_{\pi} \prod_{j=1}^{n} u_{t}\left(v_{\pi, j}\right)\right)^{2},
$$

where $y_{i}=x_{i}-x_{i-1}$,

$$
v_{\pi, j}=x_{\pi(j)}-x_{\pi(j-1)}=\sum_{k \in] \pi(j-1), \pi(j)]} y_{k},
$$


and (with a slight abuse of notation), $k \in] \pi(j-1), \pi(j)]$ means

$$
k \in] \min (\pi(j-1), \pi(j)), \max (\pi(j-1), \pi(j))] .
$$

In view of (2.20), in order to prove our lemma it suffices to show that

$$
\begin{aligned}
& n ! \sum_{y_{1}, \ldots, y_{n}}\left(\prod_{i=1}^{n} u_{t}\left(y_{i}\right)\right)\left(\sum_{\pi} \prod_{j=1}^{n} u_{t}\left(v_{\pi, j}\right)\right)\left(\sum_{\pi^{\prime}} \prod_{j=1}^{n} u_{t}\left(v_{\pi^{\prime}, j}\right)\right) \\
& =(1+\epsilon) \Psi(n) h^{n}(t)+R(n, t)
\end{aligned}
$$

with $R(n, t)$ as in (2.19). For each permutations $\sigma$ of $\{1,2, \ldots, n\}$ we define

$$
\Delta_{\sigma}=\left\{\left(y_{1}, \cdots, y_{n}\right)|| y_{\sigma(1)}|\leq| y_{\sigma(2)}|\leq \cdots \leq| y_{\sigma(n)} \mid\right\}
$$

and rewrite the left hand side of $(2.22)$ as

$$
n ! \sum_{\sigma, \pi, \pi^{\prime}} \sum_{\Delta_{\sigma}}\left(\prod_{i=1}^{n} u_{t}\left(y_{i}\right)\right)\left(\prod_{j=1}^{n} u_{t}\left(v_{\pi, j}\right)\right)\left(\prod_{k=1}^{n} u_{t}\left(v_{\pi^{\prime}, k}\right)\right),
$$

Note that by $(2.7)$

$$
\begin{aligned}
& \sum_{y \leq|x| \leq 9 y}\left(u_{t}(x)\right)^{3} \\
& \quad \leq \sum_{y \leq|x| \leq 9 y} C \frac{1}{1+|x|^{3}} \\
& \quad \leq C(\log 9 y-\log y)=C \log (9) .
\end{aligned}
$$

and that by (2.2)

$$
\sum_{x} u_{t}^{3}(x)=h(t)
$$

Let $A_{\sigma, k}=\left\{\left(y_{1}, \ldots, y_{n}\right)|| y_{\sigma_{k-1}}|\leq| y_{\sigma_{k}}|\leq 9| y_{\sigma_{k-1}} \mid\right\}$. Using Hölder's inequality, (2.24) and (2.25) we have

$$
\begin{aligned}
& \sum_{\left(y_{1}, \ldots, y_{n}\right) \in A_{\sigma, k}}\left(\prod_{i=1}^{n} u_{t}\left(y_{i}\right)\right)\left(\prod_{j=1}^{n} u_{t}\left(v_{\pi, j}\right)\right)\left(\prod_{k=1}^{n} u_{t}\left(v_{\pi^{\prime}, k}\right)\right) \\
& \leq\left(\sum_{\left(y_{1}, \ldots, y_{n}\right) \in A_{\sigma, k}} \prod_{i=1}^{n}\left(u_{t}\left(y_{i}\right)\right)^{3}\right)^{1 / 3} h^{2 n / 3}(t) \\
& \leq C h^{n-1 / 3}(t)
\end{aligned}
$$


Set

$$
\hat{\Delta}_{\sigma}=\left\{\left(y_{1}, \cdots, y_{n}\right)|9| y_{\sigma(k-1)}|<| y_{\sigma(k)} \mid, \forall k\right\} .
$$

Using (2.26) we see that the sum in (2.23) differs from the sum over $\hat{\Delta}_{\sigma}$ by an error term which can be incorporated into $R(n, t)$. Up to the error terms described above, we can write the sum in (2.23) as

$$
n ! \sum_{\sigma, \pi, \pi^{\prime}} \sum_{\left(y_{1}, \ldots, y_{n}\right) \in \hat{\Delta}_{\sigma}}\left(\prod_{i=1}^{n} u_{t}\left(y_{i}\right)\right)\left(\prod_{j=1}^{n} u_{t}\left(v_{\pi, j}\right)\right)\left(\prod_{k=1}^{n} u_{t}\left(v_{\pi^{\prime}, k}\right)\right) .
$$

Note that on $\hat{\Delta}_{\sigma}$

$$
\sum_{j=1}^{k-1}\left|y_{\sigma(j)}\right| \leq\left(\sum_{j=1}^{k-1}(1 / 9)^{k-j}\right)\left|y_{\sigma(k)}\right| \leq(1 / 8)\left|y_{\sigma(k)}\right|
$$

For given $\sigma, \pi$ define the map $\phi=\phi_{\sigma, \pi}:\{1,2, \ldots, n\} \mapsto\{1,2, \ldots, n\}$ by

$$
\phi(j)=\sigma\left(k_{\sigma, \pi, j}\right)
$$

where

$$
\left.\left.k_{\sigma, \pi, j}=\max \{k \mid \sigma(k) \in] \pi(j-1), \pi(j)\right]\right\} .
$$

Note that on $\hat{\Delta}_{\sigma}, \phi(j)$ is the unique integer in $\left.] \pi(j-1), \pi(j)\right]$ such that $\left|y_{\phi(j)}\right|=\sup _{k \in] \pi(j-1), \pi(j)]}\left|y_{k}\right|$. Futhermore, on $\hat{\Delta}_{\sigma}$, we see from (2.28) that $\frac{1}{2}\left|v_{\pi, j}\right|<\left|y_{\phi(j)}\right|<2\left|v_{\pi, j}\right|$. Using Hölder's inequality, and the bounds (2.7), (2.17) we have

$$
\begin{aligned}
& \sum_{\left(y_{1}, \ldots, y_{n}\right) \in \hat{\Delta}_{\sigma}}\left(\prod_{i=1}^{n} u_{t}\left(y_{i}\right)\right)\left(\prod_{j=1}^{n} u_{t}\left(v_{\pi, j}\right)\right)\left(\prod_{k=1}^{n} u_{t}\left(v_{\pi^{\prime}, k}\right)\right) \\
& \leq\left(\sum_{\substack{\left(y_{1}, \ldots, y_{n}\right) \in \hat{\Delta}_{\sigma} \\
\hat{A}_{j}=1}}^{n}\left(u_{t}\left(v_{\pi, j}\right)\right)^{3}\right)^{1 / 3} h^{2 n / 3}(t) \\
& \leq\left(\sum_{\substack{\left(y_{1}, \ldots, y_{n}\right) \in \hat{\Delta}_{\sigma} \\
\left|v_{\pi, j}\right| \leq t^{4}, \forall j}} \prod_{j=1}^{n}\left(u_{t}\left(v_{\pi, j}\right)\right)^{3}\right)^{1 / 3} h^{2 n / 3}(t)+C n h^{n-1 / 3}(t) \\
& \leq C\left(\sum_{\substack{\left(y_{1}, \ldots, y_{n}\right) \in \hat{\Delta}_{\sigma} \\
\left|y_{j}\right| \leq 2 t^{4}, \forall j}} \prod_{j=1}^{n} \frac{1}{1+\left|y_{\phi(j)}\right|^{3}}\right)^{1 / 3} h^{2 n / 3}(t)+C n h^{n-1 / 3}(t) .
\end{aligned}
$$


We now show that

$$
\sum_{\substack{\left(y_{1}, \ldots, y_{n}\right) \in \hat{\Delta}_{\sigma} \\\left|y_{j}\right| \leq 2 t^{4}, \forall j}} \prod_{j=1}^{n} \frac{1}{1+\left|y_{\phi(j)}\right|^{3}} \leq C h^{n-1}(t) .
$$

unless $\phi=\phi_{\sigma, \pi}:\{1,2, \ldots, n\} \mapsto\{1,2, \ldots, n\}$ is bijective.

To begin, we note that by $(2.21)$ both $\left\{y_{j}, j=1, \ldots, n\right\}$ and $\left\{v_{\pi, j}, j=\right.$ $1, \ldots, n\}$ generate $\left\{x_{j}, j=1, \ldots, n\right\}$ in the sense of linear combinations, so that both sets consist of $n$ linearly independent vectors. Furthermore, from (2.21) we see that each $v_{\pi, j}$ is a sum of vectors from $\left\{y_{j}, j=1, \ldots, n\right\}$. However, from the definitions, we see that when we write out any vector in $\left\{v_{\pi, j} \mid k_{\sigma, \pi, j} \leq m\right\}$ as such a sum, the sum will only involve vectors from $\left\{y_{\sigma(j)} \mid j \leq m\right\}$. Hence $\left\{v_{\pi, j} \mid k_{\sigma, \pi, j} \leq m\right\}$ will contain at most $m$ linearly independent vectors. Therefore, for each $m=0,1, \ldots, n-1$, the set $\left\{v_{\pi, j} \mid k_{\sigma, \pi, j}>m\right\}$ will contain at least $n-m$ elements. Equivalently, for each $m=0,1, \ldots, n-1$, the set $\left\{j \mid \sigma^{-1} \phi(j)>m\right\}$ will contain at least $n-m$ elements. This shows that for each $m=0,1, \ldots, n-1$, the product

$$
\prod_{j=1}^{n} \frac{1}{1+\left|y_{\phi(j)}\right|^{3}}
$$

will contain at least $n-m$ factors of the form

$$
\frac{1}{1+\left|y_{\sigma(j)}\right|^{3}}
$$

with $j>m$. We now return to (2.30) and sum in turn over the variables $y_{\sigma(n)}, y_{\sigma(n-1)}, \ldots, y_{\sigma(1)}$ using the fact that

$$
\sum_{\left\{y_{\sigma(j)} \in \mathbf{Z}^{3} \mid\right.} \frac{1}{\left.9\left|y_{\sigma(j-1)}\right| \leq\left|y_{\sigma(j)}\right| \leq 2 t^{4}\right\}} \frac{1}{1+\left|y_{\sigma(j)}\right|^{3}} \leq C h(t)
$$

while for any $k>1$

$$
\sum_{\left\{y_{\sigma(j)} \in \mathbf{Z}^{3} \mid\right.} \sum_{\left.9\left|y_{\sigma(j-1)}\right| \leq\left|y_{\sigma(j)}\right| \leq 2 t^{4}\right\}} \frac{1}{1+\left|y_{\sigma(j)}\right|^{3 k}} \leq C \frac{1}{1+\left|y_{\sigma(j-1)}\right|^{3(k-1)}} .
$$

The above considerations show that as we sum successively over the variables $y_{\sigma(n)}, y_{\sigma(n-1)}, \ldots, y_{\sigma(1)}$, at the stage when we sum over $y_{\sigma(j)}$, we will be 
summing a factor of the form $\frac{1}{1+\left|y_{\sigma(j)}\right|^{3 k}}$ for some $k \geq 1$, while if $\phi=\phi_{\sigma, \pi}$ : $\{1,2, \ldots, n\} \mapsto\{1,2, \ldots, n\}$ is not bijective we must have $k>1$ at some stage. These considerations, together with (2.31) and (2.32) establish (2.30), and similarly for $\phi_{\sigma, \pi^{\prime}}$.

Let $\Omega_{n}$ be the set of $\left(\sigma, \pi, \pi^{\prime}\right)$ for which $\phi_{\sigma, \pi}$ and $\phi_{\sigma, \pi^{\prime}}$ are both bijections. Up to the error terms described above, we can write the sum in (2.27) as

$$
n ! \sum_{\left(\sigma, \pi, \pi^{\prime}\right) \in \Omega_{n}} \sum_{\left(y_{1}, \ldots, y_{n}\right) \in \hat{\Delta}_{\sigma}}\left(\prod_{i=1}^{n} u_{t}\left(y_{i}\right)\right)\left(\prod_{j=1}^{n} u_{t}\left(v_{\pi, j}\right)\right)\left(\prod_{k=1}^{n} u_{t}\left(v_{\pi^{\prime}, k}\right)\right)
$$

Since on $\hat{\Delta}_{\sigma}$ we have by $(2.28)$ that $\left|y_{\phi(j)}\right| \geq 8\left|v_{\pi, j}-y_{\phi(j)}\right|$, we can then replace each occurence of $v_{\pi, j}$ in $(2.33)$ by $y_{\phi(j)}$, bounding the error terms using

$$
\begin{aligned}
& \sum_{\{|x| \geq 8|a|\}}\left(u_{t}(x+a)-u_{t}(x)\right)^{3} \\
& \quad \leq C \sum_{\{|x| \geq 8|a|\}}\left(\frac{|a|^{3}}{1+|x|^{6}}+\frac{1}{1+|x|^{4}}\right) \leq C
\end{aligned}
$$

which comes from (2.17) and Lemma 5 of the Appendix.

Thus, up to error terms described which can be incorporated into $R(n, t)$, we can write the sum in (2.33) as

$$
n ! \sum_{\left(\sigma, \pi, \pi^{\prime}\right) \in \Omega_{n}} \sum_{\left(y_{1}, \ldots, y_{n}\right) \in \hat{\Delta}_{\sigma}}\left(\prod_{i=1}^{n} u_{t}^{3}\left(y_{i}\right)\right) .
$$

Proceeding as above, up to the error terms described above, we can replace (2.35) by

$$
n ! \sum_{\left(\sigma, \pi, \pi^{\prime}\right) \in \Omega_{n}} \sum_{\left(y_{1}, \ldots, y_{n}\right) \in \Delta_{\sigma}}\left(\prod_{i=1}^{n} u_{t}^{3}\left(y_{i}\right)\right) .
$$

Since

$$
n ! \sum_{\left(y_{1}, \ldots, y_{n}\right) \in \Delta_{\sigma}}\left(\prod_{i=1}^{n} u_{t}^{3}\left(y_{i}\right)\right) \sim h^{n}(t),
$$

and as by the remark following Lemma 2.5 in [4], we have $\left|\Omega_{n}\right|=\Psi(n)$, the lemma is proved. 
We will use $E^{v, w, z}$ to denote expectation with respect to the random walks $X, X^{\prime}, X^{\prime \prime}$ where $X_{0}=v, X_{0}^{\prime}=w$ and $X_{0}^{\prime \prime}=z$. We define

$$
a(v, w, z, t)=\frac{h(v, w, z, t)}{h(t)}
$$

where

$$
\begin{aligned}
h(v, w, z, t) & =E^{v, w, z}\left(I_{t}\right) \\
2.38) & =\sum_{x \in \mathbf{Z}^{3}}\left\{\left(\sum_{i=1}^{t} p_{i}(x-v)\right)\left(\sum_{j=1}^{t} p_{j}(x-w)\right)\left(\sum_{k=1}^{t} p_{k}(x-z)\right)\right\}
\end{aligned}
$$

We will need the following lower bound.

Lemma 2 For all integers $n, t \geq 0$ and for any $\epsilon>0$

$$
E^{v, w, z}\left(I_{t}^{n}\right) \geq(1-\epsilon) \Psi(n) a(v, w, z, t / n) h^{n}(t / n)-R^{\prime}(n, t)
$$

where

$$
0 \leq R^{\prime}(n, t) \leq C(n !)^{5} h^{n-1 / 3}(t)
$$

Proof of Lemma 2: We first note that as in (2.14)

$$
\begin{gathered}
E^{v, w, z}\left(I_{t}^{n}\right) \\
\geq n ! \sum_{x_{1}, \ldots, x_{n}}\left(\prod_{i=1}^{n} u_{t / n}\left(x_{i}-x_{i-1}\right)\right)\left(\sum_{\pi} \prod_{j=1}^{n} u_{t / n}\left(x_{\pi(j)}-x_{\pi(j-1)}\right)\right) \\
\left(\sum_{\pi^{\prime}} \prod_{j=1}^{n} u_{t / n}\left(x_{\pi^{\prime}(j)}-x_{\pi^{\prime}(j-1)}\right)\right)
\end{gathered}
$$

where now we use the convention $x_{0}=v, x_{\pi(0)}=w, x_{\pi^{\prime}(0)}=z$. We then use (2.22), observing that if $\phi_{\sigma, \pi}$ is bijective we must have $\phi_{\sigma, \pi}(j)=1$ for some $j$ and this must be $j=1$ since $1 \in] \pi(j-1), \pi(j)]$ is possible only for $j=1$. Thus, $v_{\pi, 1}$ is replaced in (2.27) by $y_{1}$, and a similar analysis applies to $v_{\pi^{\prime}, 1}$.

Lemma 3 For all $t \geq 0$ and $x=O(\log \log h(t))$ we have

$$
P\left(\frac{I_{t}}{4 h(t)} \geq x\right) \leq C \sqrt{x} e^{-x} .
$$


Proof of Lemma 3: We first note that if $n=O(\log \log h(t))$ then

$$
\frac{(n !)^{5}}{h^{1 / 3}(t)} \rightarrow 0
$$

as $t \rightarrow \infty$, so that by Lemma 1 we have

$$
E\left(I_{t}^{n}\right) \leq C \Psi(n) h^{n}(t) .
$$

Then Chebyshev's inequality gives us

$$
P\left(\frac{I_{t}}{4 h(t)} \geq x\right) \leq C \frac{\Psi(n)}{(4 x)^{n}}=C \frac{\sqrt{n} n^{n} e^{-n}}{x^{n}}(1+O(1 / n))
$$

for any $n=O(\log \log h(t))$. Taking $n=[x]$ then yields $(2.42)$. $\square$.

Lemma 4 For all $\epsilon>0$ there exists an $x_{0}$ and $a t^{\prime}=t^{\prime}\left(\epsilon, x_{0}\right)$ such that for all $t \geq t^{\prime}$ and $x_{0} \leq x=O(\log \log h(t))$ we have

$$
P\left(\frac{I_{t}}{4 h(t)} \geq(1-\epsilon) x\right) \geq C_{\epsilon} e^{-x}
$$

and

$$
P^{v, w, z}\left(\frac{I_{t}}{4 h(t)} \geq(1-\epsilon) x\right) \geq C_{\epsilon}\left(a(v, w, z, 2 t /(3 x)) e^{-x}-e^{-\left(1+\epsilon^{\prime}\right) x}\right)
$$

for some $\epsilon^{\prime}>0$.

Proof of Lemma 4: This follows from Lemmas 2, 3 and (2.43) by the methods used in the proof of Lemma 2.7 in [7].

Proof of Theorem 1: For $\theta>1$ we define the sequence $\left\{t_{n}\right\}$ by

$$
h\left(t_{n}\right)=\theta^{n} .
$$

By Lemma 3 we have that for all integers $n \geq 2$ and all $\epsilon>0$

$$
P\left(\frac{I_{t_{n}}}{4 h\left(t_{n}\right) \log \log h\left(t_{n}\right)} \geq(1+\epsilon)\right) \leq C e^{-(1+\epsilon) \log n} .
$$


Therefore, by the Borel-Cantelli lemma

$$
\limsup _{n \rightarrow \infty} \frac{I_{t_{n}}}{4 h\left(t_{n}\right) \log \log h\left(t_{n}\right)} \leq 1+\epsilon \quad \text { a.s. }
$$

By taking $\theta$ arbitrarily close to 1 it is simple to interpolate in (2.50) to obtain

$$
\limsup _{n \rightarrow \infty} \frac{I_{n}}{4 h(n) \log \log h(n)} \leq 1+\epsilon \quad \text { a.s. }
$$

We now show that for any $\epsilon>0$

$$
\limsup _{n \rightarrow \infty} \frac{I_{t_{n}}}{4 h\left(t_{n}\right) \log \log h\left(t_{n}\right)} \geq 1-\epsilon \quad \text { a.s. }
$$

for all $\theta$ sufficiently large. It is sufficient to show that

$$
\limsup _{n \rightarrow \infty} \frac{I_{t_{n}}-I_{t_{n-1}}}{4 h\left(t_{n}\right) \log \log h\left(t_{n}\right)} \geq 1-\epsilon \quad \text { a.s. }
$$

Let $s_{n}=t_{n}-t_{n-1}$ and note that, as in (2.60) of [7], we have $h\left(s_{n}\right) \sim h\left(t_{n}\right)$. We also note that

$$
\left|I_{t_{n}}-I_{t_{n-1}}-I_{s_{n}} \circ \Theta_{t_{n-1}}\right| \leq I_{t_{n}, t_{n}, t_{n-1}}+I_{t_{n}, t_{n-1}, t_{n}}+I_{t_{n-1}, t_{n}, t_{n}}
$$

where $\Theta_{n}$ denotes the shift on paths defined by

$$
\left(X_{i}, X_{j}^{\prime}, X_{k}\right)\left(\Theta_{n} \omega\right)=\left(X_{n+i}, X_{n+j}^{\prime}, X^{\prime \prime}{ }_{n+k}\right)(\omega)
$$

and

$$
I_{n, m, p}=\sum_{x \in \mathbf{Z}^{3}}\left\{\left(\sum_{i=1}^{n} 1_{\left\{X_{i}=x\right\}}\right)\left(\sum_{j=1}^{m} 1_{\left\{X_{j}^{\prime}=x\right\}}\right)\left(\sum_{k=1}^{p} 1_{\left\{X_{k}^{\prime \prime}=x\right\}}\right)\right\} .
$$

As in Lemma 1 , we can show that for $t \geq t^{\prime}$, and for all integers $n \geq 0$ and any $\epsilon>0$

$$
\begin{aligned}
E\left(I_{t, t, t^{\prime}}^{n}\right) \leq(1+ & \epsilon) \Psi(n) h^{2 n / 3}(t) h^{n / 3}\left(t^{\prime}\right) \\
& +O\left((n !)^{5} h^{2 n / 3}(t) h^{n / 3-1 / 3}\left(t^{\prime}\right)\right)
\end{aligned}
$$


which, as before, leads to

$$
\begin{aligned}
& \limsup _{n \rightarrow \infty} \frac{I_{t_{n}, t_{n}, t_{n-1}}}{4 h\left(t_{n}\right) \log \log h\left(t_{n}\right)} \\
& =\limsup _{n \rightarrow \infty} \frac{I_{t_{n}, t_{n}, t_{n-1}}}{4 \sqrt[3]{\theta h^{2}\left(t_{n}\right) h\left(t_{n-1}\right)} \log \log h\left(t_{n}\right)} \\
& \leq \frac{1+\epsilon}{\sqrt[3]{\theta}} \quad \text { a.s. }
\end{aligned}
$$

Using this for $\theta$ large, (2.54), Levy's Borel-Cantelli lemma (see Corollary 5.29 in [1]) and the Markov property, we see that (2.53) will follow from

$(2.58) \sum_{n=1}^{\infty} P^{X_{t_{n-1}}, X_{t_{n-1}}^{\prime}, X_{t_{n-1}}^{\prime \prime}}\left(\frac{I_{s_{n}}}{4 h\left(s_{n}\right) \log \log h\left(s_{n}\right)} \geq 1-\epsilon\right)=\infty \quad$ a.s.

If we apply Lemma 4 with $t=s_{n}$ and $x=\log \log s_{n}$ we see that (2.58) will follow from

$$
\sum_{n=1}^{\infty} a\left(X_{t_{n-1}}, X_{t_{n-1}}^{\prime}, X_{t_{n-1}}^{\prime \prime}, s_{n} / \log n\right) \frac{1}{n^{1-\epsilon^{\prime}}}=\infty \quad \text { a.s. }
$$

We begin by showing

$$
\sum_{n=1}^{\infty} E\left(a\left(X_{t_{n-1}}, X_{t_{n-1}}^{\prime}, X_{t_{n-1}}^{\prime \prime}, s_{n} / \log n\right)\right) \frac{1}{n^{1-\epsilon^{\prime}}}=\infty
$$

To see this we note that

$$
\begin{aligned}
E\left(a\left(X_{t}, X_{t}^{\prime}, X_{t}^{\prime \prime}, m\right)\right) \\
\quad=\frac{\sum_{x \in \mathbf{Z}^{3}}\left(\sum_{i=1}^{m} p_{i+t}(x)\right)^{3}}{h(m)}
\end{aligned}
$$

so that

$$
\begin{array}{r}
E\left(a\left(X_{t_{n-1}}, X_{t_{n-1}}^{\prime}, X_{t_{n-1}}^{\prime \prime}, s_{n} / \log n\right)\right) \\
=\frac{\sum_{x \in \mathbf{Z}^{d}}\left(\sum_{i=1}^{s_{n} / \log n} p_{i+t_{n-1}}(x)\right)^{3}}{h\left(s_{n} / \log n\right)}
\end{array}
$$




$$
\begin{aligned}
= & \frac{h\left(t_{n-1}+s_{n} / \log n\right)-h\left(t_{n-1}\right)}{h\left(s_{n} / \log n\right)} \\
- & \frac{3 \sum_{x \in \mathbf{Z}^{d}}\left\{\left(\sum_{i=1}^{t_{n-1}} p_{i}(x)\right)^{2}\left(\sum_{j=1}^{\left.s_{n} / \log n\right)} p_{j+t_{n-1}}(x)\right)\right\}}{h\left(s_{n} / \log n\right)} \\
& -\frac{3 \sum_{x \in \mathbf{Z}^{d}}\left\{\left(\sum_{i=1}^{t_{n-1}} p_{i}(x)\right)\left(\sum_{j=1}^{\left.s_{n} / \log n\right)} p_{j+t_{n-1}}(x)\right)^{2}\right\}}{h\left(s_{n} / \log n\right)} .
\end{aligned}
$$

Also note that

$$
\begin{aligned}
& \frac{h\left(t_{n-1}+s_{n} / \log n\right)-h\left(t_{n-1}\right)}{h\left(s_{n} / \log n\right)} \\
& \geq \frac{h\left(s_{n} / \log n\right)-h\left(t_{n-1}\right)}{h\left(s_{n} / \log n\right)} \sim 1-\frac{1}{\theta} .
\end{aligned}
$$

This follows fairly easily since $h(t) \sim c \log (t)$. (For the details, in a more general setting, see the proof of Theorem 1.1 of [7], especially that part of the proof surrounding (2.82)). Furthermore, we have by Hölder's inequality

$$
\begin{aligned}
\frac{\sum_{x \in \mathbf{Z}^{3}}\left\{\left(\sum_{i=1}^{t_{n-1}} p_{i}(x)\right)\left(\sum_{j=1}^{\left.s_{n} / \log n\right)} p_{j+t_{n-1}}(x)\right)^{2}\right\}}{h\left(s_{n} / \log n\right)} \\
\quad \leq \frac{h^{1 / 3}\left(t_{n-1}\right) h^{2 / 3}\left(t_{n}\right)}{h\left(s_{n} / \log n\right)} \\
\quad \sim \frac{1}{\theta^{1 / 3}} .
\end{aligned}
$$

Taking $\theta$ large establishes (2.60).

Furthermore, since $a(v, w, z, t) \leq 1$ (use (2.38) and Hölder's inequality), we see that for any $\epsilon^{\prime}<1 / 2$

$$
\sum_{n=1}^{\infty} E\left(a\left(X_{t_{n-1}}, X_{t_{n-1}}^{\prime}, X_{t_{n-1}}^{\prime \prime}, s_{n} / \log n\right) \frac{1}{n^{1-\epsilon^{\prime}}}\right)^{2}<\infty .
$$

(2.59) will now follow from the Paley-Zygmund lemma, (see e.g. Inequality II on page 8 of [2]), once we show that 


$$
\begin{gathered}
\frac{E\left(a\left(X_{t_{n-1}}, X_{t_{n-1}}^{\prime}, X_{t_{n-1}}^{\prime \prime}, s_{n} / \log n\right) a\left(X_{t_{m-1}}, X_{t_{m-1}}^{\prime}, X_{t_{m-1}}^{\prime \prime}, s_{m} / \log m\right)\right)}{E\left(a\left(X_{t_{n-1}}, X_{t_{n-1}}^{\prime}, X_{t_{n-1}}^{\prime \prime}, s_{n} / \log n\right)\right) E\left(a\left(X_{t_{m-1}}, X_{t_{m-1}}^{\prime}, X_{t_{m-1}}^{\prime \prime}, s_{m} / \log m\right)\right)} \\
\leq 1+2 \epsilon
\end{gathered}
$$

for all $\epsilon>0$, when $n>m \geq N(\epsilon)$ for some $N(\epsilon)$ sufficiently large. To prove (2.66) we begin by noting that as in (2.61)

$$
E\left(h\left(X_{t}, X_{t}^{\prime}, X_{t}^{\prime \prime}, s\right)\right)=\sum_{x \in \mathbf{Z}^{3}}\left(\sum_{i=1}^{s} p_{i+t}(x)\right)^{3}
$$

and for $t^{\prime}<t$

$$
\begin{aligned}
E\left(h\left(X_{t^{\prime}}, X_{t^{\prime}}^{\prime}, X_{t^{\prime}}^{\prime \prime}, s^{\prime}\right) h\left(X_{t}, X_{t}^{\prime}, X_{t}^{\prime \prime}, s\right)\right) & =\sum_{x, y, x^{\prime}, y^{\prime}, x^{\prime \prime}, y^{\prime \prime}} h\left(x, x^{\prime}, x^{\prime \prime}, s^{\prime}\right) p_{t^{\prime}}(x) p_{t^{\prime}}\left(x^{\prime}\right) p_{t^{\prime}}\left(x^{\prime \prime}\right) h\left(y, y^{\prime}, y^{\prime \prime}, s\right) \\
= & \sum_{x, x^{\prime}, x^{\prime \prime}} h\left(x, x^{\prime}, x^{\prime \prime}, s^{\prime}\right) p_{t^{\prime}}(x) p_{t^{\prime}}\left(x^{\prime}\right) p_{t^{\prime}}\left(x^{\prime \prime}\right) \\
& \cdot \sum_{u \in \mathbf{Z}^{3}}\left\{\left(\sum_{i=1}^{s} p_{i+t-t^{\prime}}(u-x)\right)\left(\sum_{j=1}^{s} p_{j+t-t^{\prime}}\left(u-x^{\prime}\right)\right)\right. \\
\leq & \left.\quad \cdot\left(\sum_{k=1}^{s} p_{k+t-t^{\prime}}\left(u-x^{\prime \prime}\right)\right)\right\} \\
= & \sum_{x \in x^{\prime}, x^{\prime \prime}} h\left(x, x^{\prime}, x^{\prime \prime}, s^{\prime}\right) p_{t^{\prime}}(x) p_{t^{\prime}}\left(x^{\prime}\right) p_{t^{\prime}}\left(x^{\prime \prime}\right) \sum_{u \in \mathbf{Z}^{3}}\left(\sum_{j=1}^{s} p_{j+t-t^{\prime}}(u)\right)^{3} \\
& \left.\sum_{i=t^{\prime}}(x)\right)_{u \in \mathbf{Z}^{3}}\left(\sum_{j=1}^{s} p_{j+t-t^{\prime}}(u)\right)^{3} .
\end{aligned}
$$

From (2.67), (2.68) we see that

$$
\begin{aligned}
& \frac{E\left(h\left(X_{t_{m-1}}, X_{t_{m-1}}^{\prime}, X_{t_{m-1}}^{\prime \prime}, s_{m} / \log m\right) h\left(X_{t_{n-1}}, X_{t_{n-1}}^{\prime}, X_{t_{n-1}}^{\prime \prime}, s_{n} / \log n\right)\right)}{E\left(h\left(X_{t_{m-1}}, X_{t_{m-1}}^{\prime}, X_{t_{m-1}}^{\prime \prime}, s_{m} / \log m\right)\right) E\left(h\left(X_{t_{n-1}}, X_{t_{n-1}}^{\prime}, X_{t_{n-1}}^{\prime \prime}, s_{n} / \log n\right)\right)} \\
& \quad \leq \frac{\sum_{u \in \mathbf{Z}^{3}}\left(\sum_{j=1}^{s_{n} / \log n} p_{j+t_{n-1}-t_{m-1}}(u)\right)^{3}}{\sum_{u \in \mathbf{Z}^{3}}\left(\sum_{j=1}^{s_{n} / \log n} p_{j+t_{n-1}}(u)\right)^{3}} .
\end{aligned}
$$


Arguing as in (2.62)-(2.64) we see that (2.66) follows. This completes the proof of Theorem 1.

\section{Proof of Theorem 3}

We begin with some moment calculations. Recall

$$
\begin{aligned}
J_{n} & =\left|X(1, n) \cap X^{\prime}(1, n) \cap X^{\prime \prime}(1, n)\right| \\
& =\sum_{x \in \mathbf{Z}^{4}} 1_{\{x \in X(1, n)\}} 1_{\left\{x \in X^{\prime}(1, n)\right\}} 1_{\left\{x \in X^{\prime \prime}(1, n)\right\}}
\end{aligned}
$$

As usual set

$$
T_{x}=\inf \left\{k \mid X_{k}=x\right\}
$$

and note that

$$
\begin{aligned}
E\left(J_{t}^{n}\right) & =E\left[\left(\sum_{x} 1_{\{x \in X(1, t)\}} 1_{\left\{x \in X^{\prime}(1, t)\right\}} 1_{\left\{x \in X^{\prime \prime}(1, t)\right\}}\right)^{n}\right] \\
& =\sum_{x_{1}, \ldots, x_{n}} E\left(\prod_{i=1}^{n} 1_{\{x \in X(1, t)\}} 1_{\left\{x \in X^{\prime}(1, t)\right\}} 1_{\left\{x \in X^{\prime \prime}(1, t)\right\}}\right) \\
& =\sum_{x_{1}, \ldots, x_{n}}\left\{E\left(\prod_{i=1}^{n} 1_{\left\{x_{i} \in X(1, t)\right\}}\right)\right\}^{3} \\
& \leq \sum_{x_{1}, \ldots, x_{n}}\left\{\sum_{\pi} P\left(T_{x_{\pi(1)}} \leq T_{x_{\pi(2)}} \leq \cdots \leq T_{x_{\pi(n)}} \leq t\right)\right\}^{3} \\
& =\sum_{x_{1}, \ldots, x_{n}}\left(P\left(T_{x_{1}} \leq T_{x_{2}} \leq \cdots \leq T_{x_{n}} \leq t\right)\right) \\
& \cdot\left(\sum_{\pi} P\left(T_{x_{\pi(1)}} \leq T_{x_{\pi(2)}} \leq \cdots \leq T_{x_{\pi(n)}} \leq t\right)\right)^{2}
\end{aligned}
$$

where $\sum_{\pi}$ runs over the set of permutations $\pi$ of $\{1,2, \ldots, n\}$. Set

$$
v_{t}(x)=P\left(T_{x} \leq t\right) .
$$

Then we see from (3.2) that

$$
\begin{aligned}
E\left(J_{t}^{n}\right) \leq n ! & \sum_{x_{1}, \ldots, x_{n}}\left(\prod_{i=1}^{n} v_{t}\left(x_{i}-x_{i-1}\right)\right) \\
& \left(\sum_{\pi} \prod_{j=1}^{n} v_{t}\left(x_{\pi(j)}-x_{\pi(j-1)}\right)\right)^{2},
\end{aligned}
$$


while

$$
\begin{aligned}
E\left(J_{t}^{n}\right) \geq n ! \sum_{x_{1}, \ldots, x_{n}} & \left(\prod_{i=1}^{n} v_{t / n}\left(x_{i}-x_{i-1}\right)\right) \\
& \left(\sum_{\pi} \prod_{j=1}^{n} v_{t / n}\left(x_{\pi(j)}-x_{\pi(j-1)}\right)\right)^{2} .
\end{aligned}
$$

Let

$$
f_{r}(x)=P\left(T_{x}=r\right)
$$

so that

$$
v_{t}(x)=\sum_{r=1}^{t} f_{r}(x) .
$$

We have

$$
p_{j}(x)=\sum_{i=1}^{j} f_{i}(x) p_{j-i}(0)
$$

where as usual we set $p_{0}(x)=1_{\{x=0\}}$. From this we see that

$$
\begin{aligned}
u_{t}(x) & =\sum_{j=1}^{t} p_{j}(x) \\
& =\sum_{j=1}^{t} \sum_{i=1}^{j} f_{i}(x) p_{j-i}(0) \\
& =\sum_{i=1}^{t} \sum_{j=i}^{t} f_{i}(x) p_{j-i}(0) \\
& =\sum_{i=1}^{t} f_{i}(x)\left(1+u_{t-i}(0)\right) .
\end{aligned}
$$

Consequently we have

$$
u_{t}(x) \leq v_{t}(x)\left(1+u_{t}(0)\right)
$$

and

$$
u_{2 t}(x) \geq v_{t}(x)\left(1+u_{t}(0)\right) .
$$

Now it is well known that

$$
\frac{1}{1+u_{t}(0)} \downarrow q
$$


so that for any $\epsilon>0$ we can find $t_{0}<\infty$ such that

$$
q u_{t}(x) \leq v_{t}(x) \leq(q+\epsilon) u_{2 t}(x)
$$

for all $t \geq t_{0}$ and $x$. Hence (3.3) and (3.4) give us

$$
\begin{array}{r}
E\left(J_{t}^{n}\right) \leq(q+\epsilon)^{2 n} n ! \sum_{x_{1}, \ldots, x_{n}}\left(\prod_{i=1}^{n} u_{2 t}\left(x_{i}-x_{i-1}\right)\right) \\
\left(\sum_{\pi} \prod_{j=1}^{n} u_{2 t}\left(x_{\pi(j)}-x_{\pi(j-1)}\right)\right)^{2},
\end{array}
$$

and

$$
\begin{aligned}
E\left(J_{t}^{n}\right) \geq q^{2 n} n ! & \sum_{x_{1}, \ldots, x_{n}}\left(\prod_{i=1}^{n} u_{t / n}\left(x_{i}-x_{i-1}\right)\right) \\
& \left(\sum_{\pi} \prod_{j=1}^{n} u_{t / n}\left(x_{\pi(j)}-x_{\pi(j-1)}\right)\right)^{2} .
\end{aligned}
$$

The proof of Theorem 3 now follows exactly along the lines of the proof of Theorem 1.

\section{Appendix}

Lemma 5 Let $X_{n}$ be a mean-zero adapted random walk in $Z^{3}$. Assume that $E\left(\left|X_{1}\right|^{2} \log _{+}\left|X_{1}\right|\right)<\infty$. Then for some $C<\infty$

$$
|u(x+a)-u(x)| \leq \frac{C|a|}{1+|x|^{2}}
$$

for all $a, x$ satisfying $|a| \leq|x| / 8$.

Furthermore, for some $C<\infty$

$$
\left|u_{t}(x+a)-u_{t}(x)\right| \leq \frac{C|a|}{1+|x|^{2}},
$$

for all $a, x, t$ satisfying $|a| \leq|x| / 8$ and $|x|^{1 / 4}<t$. 
In [3], Lawler shows that the usual Green's function asymptotics do not necessarily hold for all mean zero finite variance random walks on $Z^{4}$. We expect that a similar analysis would show that finite variance is not enough to guarantee (4.1). Our Lemma says that $E\left(\left|X_{1}\right|^{2} \log _{+}\left|X_{1}\right|\right)<\infty$ is sufficient.

Proof of Lemma 5: Let

$$
\phi(p)=E\left(e^{i p X_{1}}\right)
$$

denote the characteristic function of $X_{1}$. We have

$$
u(x)=\frac{1}{(2 \pi)^{3 / 2}} \int_{[-\pi, \pi]^{3}} \frac{e^{i p x}}{1-\phi(p)} d p .
$$

Let $Q=\left\{Q_{i, j}\right\}$ denote the covariance matrix of $X_{1}=\left(X_{1}^{(1)}, X_{1}^{(2)}, X_{1}^{(3)}\right)$, i.e. $Q_{i, j}=E\left(X_{1}^{(i)} X_{1}^{(j)}\right)$. We write

$$
Q(p)=\frac{1}{2} \sum_{i, j=1}^{3} Q_{i, j} p_{i} p_{j} .
$$

Using our assumption that $E\left(X_{1}\right)=0$ and $E\left(\left|X_{1}\right|^{2} \log _{+}\left|X_{1}\right|\right)<\infty$, we observe that for $|p| \leq 1$

$$
\begin{aligned}
\mid 1- & \phi(p)-Q(p) \mid \\
& =\left|E\left(1-e^{i p \cdot X_{1}}+i p \cdot X_{1}+(1 / 2)(i p \cdot X)^{2}\right)\right| \\
& \leq c|p|^{3} E\left(1_{\left\{\left|X_{1}\right| \leq 1 /|p|\right\}}\left|X_{1}\right|^{3}\right)+c|p|^{2} E\left(1_{\left\{\left|X_{1}\right|>1 /|p|\right\}}\left|X_{1}\right|^{2}\right) \\
& \leq c|p|^{2} / \log _{+}(1 /|p|) \\
& =o\left(|p|^{2}\right)
\end{aligned}
$$

Similarly, we have

$$
\begin{aligned}
\left|\phi_{1}(p)+Q_{1}\right| & =\left|E\left(i X_{1}^{(1)} e^{i p \cdot X_{1}}+X_{1}^{(1)} p \cdot X_{1}\right)\right| \\
& \leq\left|E\left(i X_{1}^{(1)}\left(e^{i p \cdot X_{1}}-1-i p \cdot X_{1}\right)\right)\right| \\
& \leq c|p|^{2} E\left(1_{\left\{\left|X_{1}\right| \leq 1 /|p|\right\}}\left|X_{1}\right|^{3}\right)+c|p| E\left(1_{\left\{\left|X_{1}\right|>1 /|p|\right\}}\left|X_{1}\right|^{2}\right) \\
& =o(|p|)
\end{aligned}
$$

and

$$
\begin{aligned}
\left|\phi_{1,1}(p)+Q_{1,1}\right| & =\left|E\left(-\left(X_{1}^{(1)}\right)^{2}\left(e^{i p \cdot X_{1}}-1\right)\right)\right| \\
& \leq c|p| E\left(1_{\left\{\left|X_{1}\right| \leq 1 /|p|\right\}}\left|X_{1}\right|^{3}\right)+c E\left(1_{\left\{\left|X_{1}\right|>1 /|p|\right\}}\left|X_{1}\right|^{2}\right) \\
& =o\left(1_{|p|}\right)
\end{aligned}
$$


Using the third line of (4.4) we see that

$$
\begin{aligned}
& \int_{|p| \leq 1} \frac{|1-\phi(p)-Q(p)|}{|p|^{5}} d p \\
& \quad \leq c E\left(\left(\int_{\left\{|p| \leq 1 /\left|X_{1}\right|\right\}} \frac{1}{|p|^{2}} d p\right)\left|X_{1}\right|^{3}\right)+c E\left(\left(\int_{\left\{|p|>1 /\left|X_{1}\right|\right\}} \frac{1}{|p|^{3}} d p\right)\left|X_{1}\right|^{2}\right) \\
& \quad \leq c E\left(\left|X_{1}\right|^{2} \log _{+}\left|X_{1}\right|\right)<\infty
\end{aligned}
$$

Similarly, using (4.5) and (4.6) we see that

$$
\int_{|p| \leq 1} \frac{\left|\phi_{1}(p)+Q_{1}\right|}{|p|^{4}} d p<\infty
$$

and

$$
\int_{|p| \leq 1} \frac{\left|\phi_{1,1}(p)+Q_{1,1}\right|}{|p|^{3}} d p<\infty
$$

Let $q_{t}(x)$ denote the transition density for Brownian motion in $R^{3}$ and set

$$
v_{\delta}(x)=\int_{\delta}^{\infty} q_{t}(x) d t=\frac{1}{(2 \pi)^{3 / 2}} \int_{R^{3}} e^{i p x} \frac{e^{-\delta|p|^{2} / 2}}{|p|^{2} / 2} d p .
$$

We have

$$
\frac{v_{\delta}\left(Q^{-1 / 2} x\right)}{|Q|^{1 / 2}}=\frac{1}{(2 \pi)^{3 / 2}} \int_{R^{3}} e^{i p x} \frac{e^{-\delta Q(p)}}{Q(p)} d p .
$$

Note that

$$
v_{\delta}(x) \uparrow v_{0}(x)=\int_{0}^{\infty} q_{t}(x) d t=\frac{1}{2 \pi|x|}
$$

as $\delta \rightarrow 0$.

If $x=\left(x_{1}, x_{2}, x_{3}\right)$, we can assume, without loss of generality, that $|x| \neq 0$ and that $\left|x_{1}\right|=\max _{j}\left|x_{j}\right|$. We have

$$
\begin{aligned}
& \frac{v_{\delta}\left(Q^{-1 / 2} x\right)}{|Q|^{1 / 2}}=\frac{1}{(2 \pi)^{3 / 2}} \int_{A} e^{i p x} \frac{e^{-\delta Q(p)}}{Q(p)} d p \\
& \quad+\frac{1}{(2 \pi)^{3 / 2}} \int_{B} e^{i p x} \frac{e^{-\delta Q(p)}}{Q(p)} d p+\frac{1}{(2 \pi)^{3 / 2}} \int_{C} e^{i p x} \frac{e^{-\delta Q(p)}}{Q(p)} d p
\end{aligned}
$$

where $A=[-\pi, \pi]^{3}, B=[-\pi, \pi]^{c} \times[-\pi, \pi]^{2}$, and $C=R \times\left([-\pi, \pi]^{2}\right)^{c}$. Note that

$$
C=\left\{\left|p_{2}\right|>\pi\right\} \bigcup\left\{\left|p_{3}\right|>\pi\right\}
$$


We have

$$
\begin{aligned}
u(x+a) & -u(x)-\left(\frac{v_{\delta}\left(Q^{-1 / 2}(x+a)\right)}{|Q|^{1 / 2}}-\frac{v_{\delta}\left(Q^{-1 / 2} x\right)}{|Q|^{1 / 2}}\right) \\
= & \frac{1}{(2 \pi)^{3 / 2}} \int_{A}\left(e^{i p(x+a)}-e^{i p x}\right)\left(\frac{1}{1-\phi(p)}-\frac{e^{-\delta Q(p)}}{Q(p)}\right) d p \\
& -\frac{1}{(2 \pi)^{3 / 2}} \int_{B}\left(e^{i p(x+a)}-e^{i p x}\right) \frac{e^{-\delta Q(p)}}{Q(p)} d p \\
& -\frac{1}{(2 \pi)^{3 / 2}} \int_{C}\left(e^{i p(x+a)}-e^{i p x}\right) \frac{e^{-\delta Q(p)}}{Q(p)} d p
\end{aligned}
$$

To prove (4.1) it suffices to show that in the limit as $\delta \rightarrow 0$ the right hand side is $O\left(|a| /|x|^{2}\right)$.

We first show that

$$
\lim _{\delta \rightarrow 0}\left|\int_{C} e^{i p x} \frac{e^{-\delta Q(p)}}{Q(p)} d p\right| \leq \frac{c}{|x|^{2}} .
$$

To see this we integrate by parts twice in the $p_{1}$ direction to get

$$
\int_{C} e^{i p x} \frac{e^{-\delta Q(p)}}{Q(p)} d p=\frac{i^{2}}{x_{1}^{2}} \int_{C} e^{i p x} D_{1}^{2}\left(\frac{e^{-\delta Q(p)}}{Q(p)}\right) d p
$$

and

$$
\begin{aligned}
& D_{1}^{2}\left(\frac{e^{-\delta Q(p)}}{Q(p)}\right)=D_{1}^{2}\left(e^{-\delta Q(p)}\right) \frac{1}{Q(p)}+2 D_{1}\left(e^{-\delta Q(p)}\right) D_{1}\left(\frac{1}{Q(p)}\right) \\
& \quad+e^{-\delta Q(p)} D_{1}^{2}\left(\frac{1}{Q(p)}\right)
\end{aligned}
$$

Note that $\inf _{p \in B \bigcup C} Q(p) \geq d>0$. Also, $D_{1}^{j}\left(\frac{1}{Q(p)}\right)$ is homogeneous in $p$ of degree $-(2+j)$, so that the last term in (4.18) is integrable on $C$ even when we take $\delta=0$. Since

$$
D_{1}\left(e^{-\delta Q(p)}\right)=-\delta Q_{1}(p) e^{-\delta Q(p)}
$$

and $Q_{1}(p) D_{1}\left(\frac{1}{Q(p)}\right)$ is homogeneous in $p$ of degree -2 , scaling out $\delta$ shows that the integral of the absolute value of the second term in (4.18) is bounded by

$$
\delta^{1 / 2} \int \frac{e^{-Q(p)}}{|p|^{2}} d p \leq c \delta^{1 / 2} .
$$


The first term in (4.18) is handled similarly, proving (4.16).

For the first two integrals on the right hand side of (4.15) we integrate by parts in the $p_{1}$ direction to obtain

$$
\begin{gathered}
\frac{i}{\left(x_{1}+a_{1}\right)} \frac{1}{(2 \pi)^{3 / 2}} \int_{A} e^{i p(x+a)} D_{1}\left(\frac{1}{1-\phi(p)}-\frac{e^{-\delta Q(p)}}{Q(p)}\right) d p \\
-\frac{i}{x_{1}} \frac{1}{(2 \pi)^{3 / 2}} \int_{A} e^{i p x} D_{1}\left(\frac{1}{1-\phi(p)}-\frac{e^{-\delta Q(p)}}{Q(p)}\right) d p \\
-\frac{i}{\left(x_{1}+a_{1}\right)} \frac{1}{(2 \pi)^{3 / 2}} \int_{B} e^{i p(x+a)} D_{1}\left(\frac{e^{-\delta Q(p)}}{Q(p)}\right) d p \\
+\frac{i}{x_{1}} \frac{1}{(2 \pi)^{3 / 2}} \int_{B} e^{i p x} D_{1}\left(\frac{e^{-\delta Q(p)}}{Q(p)}\right) d p
\end{gathered}
$$

where we have used the fact that the boundary terms coming from the integrals over $A$ and $B$ cancel. (These boundary terms are easily seen to be finite).

We claim that (4.21) is equal to

$$
\begin{gathered}
\frac{i}{x_{1}} \frac{1}{(2 \pi)^{3 / 2}} \int_{A} e^{i p x}\left(e^{i p a}-1\right) D_{1}\left(\frac{1}{1-\phi(p)}-\frac{e^{-\delta Q(p)}}{Q(p)}\right) d p \\
-\frac{i}{x_{1}} \frac{1}{(2 \pi)^{3 / 2}} \int_{B} e^{i p x}\left(e^{i p a}-1\right) D_{1}\left(\frac{e^{-\delta Q(p)}}{Q(p)}\right) d p \\
+O\left(|a| /|x|^{2}\right) .
\end{gathered}
$$

To establish our last claim, using the fact that

$$
\left|\frac{1}{\left(x_{1}+a_{1}\right)}-\frac{1}{x_{1}}\right| \leq c|a| /|x|^{2},
$$

it suffices to show that

$$
\int_{A}\left|D_{1}\left(\frac{1}{1-\phi(p)}-\frac{e^{-\delta Q(p)}}{Q(p)}\right)\right| d p<\infty
$$

and

$$
\int_{B}\left|D_{1}\left(\frac{e^{-\delta Q(p)}}{Q(p)}\right)\right| d p<\infty
$$


with bounds uniform in $0<\delta \leq 1$. (4.24) is easily seen to be bounded independently of $\delta \leq 1$, using ideas similar to those we used in bounding the integral in (4.17). As for (4.23), we first observe that

$$
\begin{aligned}
& D_{1}\left(\frac{1}{1-\phi(p)}-\frac{e^{-\delta Q(p)}}{Q(p)}\right) \\
& =\left(\frac{\phi_{1}(p)}{(1-\phi(p))^{2}}+\frac{Q_{1}(p)}{(Q(p))^{2}}\right)+D_{1}\left(\frac{1-e^{-\delta Q(p)}}{Q(p)}\right)
\end{aligned}
$$

The integral of the last term is bounded easily as before. As to the first term in $(4.25)$, we write

$$
\begin{aligned}
& \frac{\phi_{1}(p)}{(1-\phi(p))^{2}}+\frac{Q_{1}(p)}{(Q(p))^{2}} \\
= & \frac{\phi_{1}(p)+Q_{1}(p)}{(1-\phi(p))^{2}}+Q_{1}(p)\left(\frac{1}{(Q(p))^{2}}-\frac{1}{(1-\phi(p))^{2}}\right) \\
= & \frac{\phi_{1}(p)+Q_{1}(p)}{(1-\phi(p))^{2}}+Q_{1}(p)\left(\frac{(1-\phi(p))^{2}-(Q(p))^{2}}{(Q(p))^{2}(1-\phi(p))^{2}}\right) \\
= & \frac{\phi_{1}(p)+Q_{1}(p)}{(1-\phi(p))^{2}} \\
& \quad+(1-\phi(p)-Q(p))\left(\frac{Q_{1}(p)(1-\phi(p)+Q(p))}{(Q(p))^{2}(1-\phi(p))^{2}}\right)
\end{aligned}
$$

The integrals of the two terms in the last equality are bounded by (4.8) and (4.7) respectively. This establishes our claim that (4.21) is equal to (4.22).

To bound the integrals in (4.22) we now integrate by parts once more in the $p_{1}$ direction to obtain

$$
\begin{gathered}
=\frac{i^{2}}{x_{1}^{2}} \frac{1}{(2 \pi)^{3 / 2}} \int_{A} e^{i p x} D_{1}\left\{\left(e^{i p a}-1\right) D_{1}\left(\frac{1}{1-\phi(p)}-\frac{e^{-\delta Q(p)}}{Q(p)}\right)\right\} d p \\
-\frac{i^{2}}{x_{1}^{2}} \frac{1}{(2 \pi)^{3 / 2}} \int_{B} e^{i p x} D_{1}\left\{\left(e^{i p a}-1\right) D_{1}\left(\frac{e^{-\delta Q(p)}}{Q(p)}\right)\right\} d p .
\end{gathered}
$$

Once again, the (finite) boundary terms cancel. (Actually, each boundary term is $O\left(1 /|x|^{2}\right)$.) Using the bounds (4.23) and (4.24), we find that (4.27) 
equals

$$
\begin{gathered}
=\frac{i^{2}}{x_{1}^{2}} \frac{1}{(2 \pi)^{3 / 2}} \int_{A} e^{i p x}\left(e^{i p a}-1\right) D_{1}^{2}\left(\frac{1}{1-\phi(p)}-\frac{e^{-\delta Q(p)}}{Q(p)}\right) d p \\
-\frac{i^{2}}{x_{1}^{2}} \frac{1}{(2 \pi)^{3 / 2}} \int_{B} e^{i p x}\left(e^{i p a}-1\right) D_{1}^{2}\left(\frac{e^{-\delta Q(p)}}{Q(p)}\right) d p \\
+O\left(|a| /|x|^{2}\right)
\end{gathered}
$$

As in the proof of (4.16), we see that

$$
\lim _{\delta \rightarrow 0}\left|\int_{B} e^{i p x}\left(e^{i p a}-1\right) D_{1}^{2}\left(\frac{e^{-\delta Q(p)}}{Q(p)}\right) d p\right| \leq c .
$$

To handle the first integral in (4.28) we note that

$$
\begin{gathered}
\left(e^{i p a}-1\right) D_{1}^{2}\left(\frac{1}{1-\phi(p)}-\frac{e^{-\delta Q(p)}}{Q(p)}\right) \\
=\left(e^{i p a}-1\right) D_{1}^{2}\left(\frac{1}{1-\phi(p)}-\frac{1}{Q(p)}\right) \\
+\left(e^{i p a}-1\right)\left(1-e^{-\delta Q(p)}\right) D_{1}^{2}\left(\frac{1}{Q(p)}\right) \\
-2\left(e^{i p a}-1\right) D_{1}\left(e^{-\delta Q(p)}\right) D_{1}\left(\frac{1}{Q(p)}\right) \\
-\left(e^{i p a}-1\right) D_{1}^{2}\left(e^{-\delta Q(p)}\right) \frac{1}{Q(p)}
\end{gathered}
$$

Once again it is easy to control the last three terms on the right hand side of (4.30), while for the first term we use

$$
\begin{aligned}
& D_{1}^{2}\left(\frac{1}{1-\phi(p)}-\frac{1}{Q(p)}\right)=\left(\frac{\phi_{1,1}(p)}{(1-\phi(p))^{2}}+\frac{Q_{1,1}}{(Q(p))^{2}}\right) \\
& \quad+2\left(\frac{\left(\phi_{1}(p)\right)^{2}}{(1-\phi(p))^{3}}-\frac{\left(Q_{1}(p)\right)^{2}}{(Q(p))^{3}}\right) .
\end{aligned}
$$

As in (4.26), we write out the first term on the right hand side of (4.31) as

$$
\frac{\phi_{1,1}(p)}{(1-\phi(p))^{2}}+\frac{Q_{1,1}}{(Q(p))^{2}}
$$




$$
\begin{aligned}
= & \frac{\phi_{1,1}(p)+Q_{1,1}}{(1-\phi(p))^{2}} \\
& +(1-\phi(p)-Q(p))\left(\frac{Q_{1,1}(1-\phi(p)+Q(p))}{(Q(p))^{2}(1-\phi(p))^{2}}\right)
\end{aligned}
$$

Hence, we can bound

$$
\begin{aligned}
\mid e^{i p a} & -1|| \frac{\phi_{1,1}(p)}{(1-\phi(p))^{2}}+\frac{Q_{1,1}}{(Q(p))^{2}} \mid \\
& \leq \frac{c|a|\left|\phi_{1,1}(p)+Q_{1,1}\right|}{|p|^{3}}+\frac{c|a||1-\phi(p)-Q(p)|}{|p|^{5}} .
\end{aligned}
$$

The integrals are now bounded using (4.6) and (4.4) respectively.

Similarly we write out the second term on the right hand side of (4.31) as

$$
\begin{aligned}
& \frac{\left(\phi_{1}(p)\right)^{2}}{(1-\phi(p))^{3}}-\frac{\left(Q_{1}(p)\right)^{2}}{(Q(p))^{3}} \\
= & \frac{\left(\phi_{1}(p)\right)^{2}-\left(Q_{1}(p)\right)^{2}}{(1-\phi(p))^{3}}+\left(Q_{1}(p)\right)^{2}\left(\frac{1}{(Q(p))^{3}}-\frac{1}{(1-\phi(p))^{3}}\right) \\
= & \frac{\left(\phi_{1}(p)\right)^{2}-\left(Q_{1}(p)\right)^{2}}{(1-\phi(p))^{3}}+\left(Q_{1}(p)\right)^{2}\left(\frac{(1-\phi(p))^{3}-(Q(p))^{3}}{(Q(p))^{3}(1-\phi(p))^{3}}\right) \\
= & \left(\phi_{1}(p)+Q_{1}(p)\right)\left(\frac{\phi_{1}(p)-Q_{1}(p)}{(1-\phi(p))^{3}}\right)+(1-\phi(p)-Q(p)) \times \\
& \quad \times\left(\frac{\left(Q_{1}(p)\right)^{2}\left((1-\phi(p))^{2}+(1-\phi(p)) Q(p)+(Q(p))^{2}\right)}{(Q(p))^{3}(1-\phi(p))^{3}}\right)
\end{aligned}
$$

and we can bound

$$
\begin{aligned}
\mid e^{i p a} & -1|| \frac{\left(\phi_{1}(p)\right)^{2}}{(1-\phi(p))^{3}}-\frac{\left(Q_{1}(p)\right)^{2}}{(Q(p))^{3}} \mid \\
& \leq \frac{c|a|\left|\phi_{1}(p)+Q_{1}(p)\right|}{|p|^{4}}+\frac{c|a||1-\phi(p)-Q(p)|}{|p|^{5}} .
\end{aligned}
$$

The integrals are now bounded using (4.5) and (4.4) respectively, completing the proof of (4.1). 
To prove (4.2) we first note that

$$
u_{n-1}(x)=\frac{1}{(2 \pi)^{3 / 2}} \int_{[-\pi, \pi]^{3}} \frac{e^{i p x}\left(1-\phi^{n}(p)\right)}{1-\phi(p)} d p .
$$

Set

$$
v_{\delta}^{n}(x)=\int_{\delta}^{n} q_{t}(x) d t=\frac{1}{(2 \pi)^{3 / 2}} \int_{R^{3}} e^{i p x} \frac{e^{-\delta|p|^{2} / 2}-e^{-n|p|^{2} / 2}}{|p|^{2} / 2} d p .
$$

We note that by the mean-value theorem

$$
\begin{aligned}
\left|q_{t}(x+a)-q_{t}(x)\right| & \leq C|a| \sup _{0 \leq \theta \leq 1} \frac{|x+\theta a|}{t} q_{t}(x+\theta a) \\
& \leq C|a| \frac{|x|}{t} q_{2 t}(x)
\end{aligned}
$$

where we have used the fact that under our assumptions

$$
\frac{1}{2}|x| \leq|x+\theta a| \leq \frac{3}{2}|x|
$$

Since $t^{-1} q_{t}(x)$ is, up to a constant multiple, the transition density for Brownian motion in $R^{5}$, which has Green's function $C|x|^{-3}$, we have

$$
\left|v_{\delta}^{n}(x+a)-v_{\delta}^{n}(x)\right| \leq C|a| \int_{0}^{\infty} \frac{|x|}{t} q_{t}(x) d t \leq C \frac{|a|}{|x|^{2}} .
$$

Therefore, it suffices to bound as before an expression of the form (4.15) where $u$ is replaced by $u_{n-1}$ and $v_{\delta}$ is replaced by $v_{\delta}^{n}$. All bounds involving $v_{\delta}^{n}$ on $B$ and $C$ are handled as before. One must verify that in each case no (divergent) factors involving $n$ will remain. For example, whereas in the bound for the second term on the right hand side of (4.18) we were satisfied with a bound $c \delta^{1 / 2}$, see (4.20), when $\delta$ is replaced with $n$ we now argue that

$$
\left|Q_{1}(p) D_{1}\left(\frac{1}{Q(p)}\right)\right| \leq \frac{c Q_{1}^{2}(p)}{Q^{2}(p)} \leq c|p|^{2}
$$

on $C$, (where $Q(p) \geq d>0$ ) and scaling out $n$ now gives us a bound of $n^{-3 / 2}$. 
The analogue of the first term on the right hand side of (4.15) is

$$
\begin{gathered}
\int_{A}\left(e^{i p(x+a)}-e^{i p x}\right)\left(\frac{1-\phi^{n}(p)}{1-\phi(p)}-\frac{e^{-\delta Q(p)}-e^{-n Q(p)}}{Q(p)}\right) d p \\
=\int_{A}\left(e^{i p(x+a)}-e^{i p x}\right)\left(\frac{1}{1-\phi(p)}-\frac{e^{-\delta Q(p)}}{Q(p)}\right) d p \\
\quad-\int_{A}\left(e^{i p(x+a)}-e^{i p x}\right)\left(\frac{\phi^{n}(p)}{1-\phi(p)}-\frac{e^{-n Q(p)}}{Q(p)}\right) d p .
\end{gathered}
$$

The first term is precisely the first term on the right hand side of (4.15), so that it only remains to bound the second term. It will be necessary to modify the preceeding proof at various stages. For example, in analogy with (4.23), let us show

$$
\int_{A}\left|D_{1}\left(\frac{\phi^{n}(p)}{1-\phi(p)}-\frac{e^{-n Q(p)}}{Q(p)}\right)\right| d p<\infty
$$

We write out

$$
\begin{aligned}
& D_{1}\left(\frac{\phi^{n}(p)}{1-\phi(p)}-\frac{e^{-n Q(p)}}{Q(p)}\right) \\
& =n\left(\frac{\phi_{1}(p) \phi^{n-1}(p)}{1-\phi(p)}+\frac{Q_{1}(p) e^{-n Q(p)}}{Q(p)}\right) \\
& +\frac{\phi_{1}(p) \phi^{n}(p)}{(1-\phi(p))^{2}}+\frac{Q_{1}(p) e^{-n Q(p)}}{Q^{2}(p)}
\end{aligned}
$$

By aperiodicity, for any $\epsilon>0$ we have that $\sup _{|p| \geq \epsilon}|\phi(p)| \leq \gamma$ for some $\gamma<1$, so that, using our assumption that $n-1>|x|^{1 / 4}$, we find that the factor $\phi^{n}(p)$ gives us rapid falloff in $|x|$, and clearly this also holds for $e^{-n Q(p)}$. In the region $|p| \leq \epsilon$ we will use (4.4)-(4.6). It is then easily seen that the integral (over $|p| \leq \epsilon)$ of the second line in (4.42) is bounded after scaling in $n$. The third line is more delicate since both $\phi_{1}(p) /(1-\phi(p))^{2}$ and $Q_{1}(p) / Q^{2}(p)$ look like $|p|^{-3}$. To handle this we write

$$
\begin{aligned}
& \frac{\phi_{1}(p) \phi^{n}(p)}{(1-\phi(p))^{2}}+\frac{Q_{1}(p) e^{-n Q(p)}}{Q^{2}(p)} \\
= & \left(\phi_{1}(p)+Q_{1}(p)\right) \frac{\phi^{n}(p)}{(1-\phi(p))^{2}}-Q_{1}(p)\left(\frac{\phi^{n}(p)}{(1-\phi(p))^{2}}-\frac{e^{-n Q(p)}}{Q^{2}(p)}\right)
\end{aligned}
$$




$$
\begin{gathered}
=\left(\phi_{1}(p)+Q_{1}(p)\right) \frac{\phi^{n}(p)}{(1-\phi(p))^{2}}-Q_{1}(p) \phi^{n}(p)\left(\frac{1}{(1-\phi(p))^{2}}-\frac{1}{Q^{2}(p)}\right) \\
-\frac{Q_{1}(p)}{Q^{2}(p)}\left(\phi^{n}(p)-e^{-n Q(p)}\right) .
\end{gathered}
$$

Using $\left|\phi^{n}(p)\right| \leq 1$, the first two terms on the right hand side are handled exactly the way we handled the second line of (4.26). As for the last term, we use (recall that $|p| \leq \epsilon$ )

$$
\begin{aligned}
& \frac{\left|Q_{1}(p)\right|}{Q^{2}(p)}\left|\phi^{n}(p)-e^{-n Q(p)}\right| \\
& \leq \frac{\left|Q_{1}(p)\right|}{Q^{2}(p)}\left|\phi(p)-e^{-Q(p)}\right| n e^{-n Q(p) / 2} .
\end{aligned}
$$

Since (4.4) shows that $\left|\phi(p)-e^{-Q(p)}\right|=O\left(|p|^{2}\right)$, the integral of this last term can also be bounded by scaling in $n$. The rest of the proof can be handled similarly.

\section{References}

1. L. Breiman, Probability, Society for Industrial and Applied Mathematics, Philadelphia, 1992.

2. J.-P. Kahane, Some random series of functions, Cambridge University Press, Cambridge, 1985.

3. G. Lawler, A note on the Green's function for random walks in four dimensions, Duke Math. Preprint, 94-03.

4. J.-F. Le Gall, Proprietes d'intersection des marches aleatoires, II, Comm. Math. Phys. 104 (1986), 509-528.

5. J.-F. Le Gall and J. Rosen, The range of stable random walks, Ann. Probab. 19 (1991), 650-705.

6. M. Marcus and J. Rosen, Laws of the iterated logarithm for intersections of random walks on $Z^{4}$, Ann. de l'I.H.P., to appear. 
7. _ Laws of the iterated logarithm for the local times of recurrent random walks on $Z^{2}$ and of Levy processes and recurrent random walks in the domain of attraction of Cauchy random variables, Ann. Inst. H. Poincaré Prob. Stat. 30 (1994), 467-499.

8. _ Laws of the iterated logarithm for the local times of symmetric Levy processes and recurrent random walks, Ann. Probab. 22 (1994), 626658.

9. F. Spitzer, Principles of random walk, Springer Verlag, New York, 1976.

Jay Rosen

Department of Mathematics

College of Staten Island, CUNY

Staten Island, NY 10314 